\title{
Influence of viral hepatitis status on prognosis in patients undergoing hepatic resection for hepatocellular carcinoma: a meta-analysis of observational studies
}

\author{
Yanming Zhou ${ }^{1}$, Xiaoying $\mathrm{Si}^{2}$, Lupeng $\mathrm{Wu}^{1}, \mathrm{Xu} \mathrm{Su}{ }^{1}$, Bin $\mathrm{Li}^{1}$ and Zhiming Zhang ${ }^{3 *}$
}

\begin{abstract}
Background: The influence of viral hepatitis status on prognosis in patients undergoing hepatic resection for hepatocellular carcinoma (HCC) remains a matter of debate. This study is a meta-analysis of the available evidence.

Methods: A literature search was performed to identify comparative studies reporting postoperative survival of HCC in different types of viral hepatitis. Pooled odds ratios (OR) and weighted mean differences (WMD with 95\% confidence intervals $(95 \% \mathrm{Cl})$ were calculated using either the fixed effects model or random effects model.

Results: Twenty studies matched the selection criteria and reported on 4744 subjects, of whom 2008 in the HBVpositive (B-HCC) group, 2222 in the HCV-positive (C-HCC) group, and 514 in the hepatitis B- and C-negative (NBNCHCC). Meta-analysis showed that patients with HBV or HCV infection had a worse 5-year disease-free survival when compared to patients with NBNC-HCC (respectively: OR: 0.39, 95\% Cl: 0.28 to 0.53, P < 0.001; WMD: 0.37, 95\% Cl: 0.22 to $0.64, \mathrm{P}<0.001$ ). There was a tendency toward higher 5 -year overall survival rates in the NBNC-HCC group compared to those in the other two groups, although these differences were not statistically significant. Both the 5-year overall survival and disease-free survival were not different among the B-HCC and C-HCC groups.
\end{abstract}

Conclusions: Patients with positive serology for hepatitis B or C undergoing resection for HCC had a poor prognosis compared to patients with negative serology.

Keywords: Hepatocellular carcinoma, Viral infection, Hepatitis B, Hepatitis C, Prognosis

\section{Background}

Hepatocellular carcinoma (HCC) is the fifth most common cancer in the world, responsible for 500,000 deaths globally every year [1]. Chronic viral hepatitis and liver cirrhosis related to hepatitis B virus (HBV) and hepatitis $\mathrm{C}$ virus (HCV) infections represent the major known risk factors for HCC. A review of the literature reveals that $75 \%$ to $80 \%$ of cases of HCC are attributable to persistent viral infections with either HBV (50\%-55\%) or HCV (25\%-30\%) [2]. Nevertheless, some patients with HCC are dually infected, whereas others are negative for both HBV and HCV [3-7].

\footnotetext{
* Correspondence: z.zhiming@yahoo.com.cn

${ }^{3}$ Cancer Center, the First affiliated Hospital of Xiamen University, Xiamen, China

Full list of author information is available at the end of the article
}

Hepatic resection is widely accepted as the treatment of choice for HCC. With regard to surgery, it is important to determine whether or not the prognosis after resection differ according to the viral status. So far, the influence of viral status on prognosis for patients with HCC treated by resection remains controversial. For example, Yamanaka et al. [3] reported that the diseasefree and overall survival rates of hepatitis B- and Cnegative group were better than those of viral infections groups. In contrast, Pawlik et al. [5] reported that the presence of viral hepatitis did not significantly affect the survival rate.

Meta-analysis can be used to evaluate the existing literature in both a qualitative and quantitative way by comparing and integrating the results of different studies and taking into account variations in characteristics that

\section{Biomed Central}


can influence the overall estimate of the outcome of interest [8]. This study uses metaanalytical techniques to evaluate the influence of viral hepatitis status on prognosis in patients with HCC treated by surgery.

\section{Methods}

\section{Study Selection}

Infection with $\mathrm{HBV}$ was defined as positivity for hepatitis B surface antigen ( $\mathrm{HBsAg}$ ) or for anti-hepatitis B core antibody. Infection with $\mathrm{HCV}$ was defined as positivity for serum anti-HCV antibody (HBcAb). Therefore, patients were divided into four groups: HBV-positive (B-HCC), HCV-positive (C-HCC), dual hepatitis $\mathrm{B}$ - and $\mathrm{C}$ positive (BC-HCC), and hepatitis B- and C-negative (NBNCHCC). A MEDLINE, EMBASE, OVID, and Cochrane database search was performed on all studies reporting postoperative survival between four groups. The following Mesh search headings were used: "hepatitis B virus," "hepatitis $C$ virus," "hepatocellular carcinoma," "survival rate," "liver resection," and "hepatectomy". Only studies on humans and in English language were considered for inclusion. Reference lists of all retrieved articles were manual searched for additional studies.

\section{Data Extraction}

Two reviewers (LW and XS, respectively) independently extracted the following parameters from each study: first author, year of publication, study population characteristics, study design, inclusion and exclusion criteria, number of patients with different preoperative viral status, male: female ratio. All relevant text, tables and figures were reviewed for data extraction. Discrepancies between the two reviewers were resolved by discussion and consensus.

\section{Criteria for Inclusion and Exclusion}

For inclusion in the meta-analysis, a study had to fulfill the following criteria: 1) evaluate the influence of viral hepatitis status on prognosis in HCC patients undergoing hepatic resection; 2) report on at least one of the outcome measures mentioned below; 3 ) In dual (or multiple) studies were reported by the same institution and/or authors, either the one of higher quality or the most recent publication was included in the analysis.

Abstracts, letters, editorials and expert opinions, reviews without original data, case reports and studies lacking control groups were excluded. The following studies were also excluded: 1) those with no clearly reported outcomes of interest; 2) those evaluating patients with other types of malignant liver tumors and did not contain a distinct group of patients with HCC; or (3) those including patients undergoing palliative treatment (noncurative surgical intent).

\section{Outcomes of Interest}

Primary outcomes of interest were 5-year overall and disease-free survival after resection. Secondary outcomes of interest were clinicopathologic features.

\section{Statistical Methods}

The meta-analysis was performed using the Review Manager (RevMan) software, version 4.2.7 (The Cochrane Collaboration, Software Update, Oxford). We analysed dichotomous variables using estimation of odds ratios (OR) with a $95 \%$ confidence interval $(95 \% \mathrm{CI})$, and continuous variables using weighted mean difference (WMD) with a 95\% CI. The overall effect was tested using $\mathrm{Z}$ scores, with significance being set at $P<0.05$. Pooled effect was calculated using either the fixed effects model or random effects model. Heterogeneity was evaluated by $\chi^{2}$ and $\mathrm{I}^{2}$. In the absence of statistically significant heterogeneity, the fixed-effect method was used to combine the results. When heterogeneity was confirmed $(P \leq 0.10)$, the random-effect method was used [9].

\section{Results}

\section{Selection of Studies}

The search strategy initially generated 38 studies [3-7,10-42]. Of these studies, 18 were excluded for various reasons: 11 including patients with unresectable lesions $[6,7,10-18]$, four without survival information [19-21]. Three were published by the same team with overlapping study populations [23-25]. Finally, a total of 20 studies published between 1995 and 2011 matched the inclusion criteria and were therefore included [3-5,27-42].

The patients with $\mathrm{BC}-\mathrm{HCC}$ were too small in number and so were not separately analyzed in many studies. Only seven of 20 studies reported 186 cases of such patients in current review [3-5,27-29]. To avoid high bias-risk of publication, we did not perform an analysis of BC-HCC group. Therefore, 4744 patients were included in the meta-analysis, of whom 2008 in the B-HCC group, 2222 in the $\mathrm{C}$-HCC group, and 514 in the NBNC-HCC group. The median or mean (range) duration for the entire cohort of patients in 11 studies providing data on followup ranged from 20.3 to 132 months. In two manuscripts, Ahmad et al. [35] and Sasaki et al. [40] reported the data of subsets of patients. The characteristics of these $20 \mathrm{stu}-$ dies are summarized in Table 1.

\section{Patients Characteristics}

Results from overall meta-analysis are outlined in Table 2.

The mean age of patients in the B-HCC group was significantly younger than that of both the C-HCC (WMD: $-10.11,95 \% \mathrm{CI}:-11.14$ to $-9.09, P<0.001$ ) and the NBNC-HCC groups (WMD: $-10.42,95 \%$ CI: -12.72 
Table 1 Baseline characteristics of studies included in the meta-analysis

\begin{tabular}{|c|c|c|c|c|c|c|c|}
\hline Author & Year & Country & Group & $\begin{array}{l}\text { No. of } \\
\text { patients }\end{array}$ & $\begin{array}{l}\text { Male/ } \\
\text { Female }\end{array}$ & $\begin{array}{l}\text { Mean age } \\
\text { (years) }\end{array}$ & $\begin{array}{l}\text { Mean follow-up } \\
\text { (months) }\end{array}$ \\
\hline Takenaka [30] & 1995 & Japan & $\begin{array}{l}\text { B-HCC } \\
\text { C-HCC }\end{array}$ & $\begin{array}{l}30 \\
96\end{array}$ & $\begin{array}{l}22 / 8 \\
77 / 19\end{array}$ & $\begin{array}{l}57.0 \pm 9.4 \\
61.7 \pm 6.9\end{array}$ & - \\
\hline Miyagawa [31] & 1996 & Japan & $\begin{array}{l}\text { B-HCC } \\
\text { C-HCC } \\
\text { NBNC }\end{array}$ & $\begin{array}{l}32 \\
124 \\
19\end{array}$ & $\begin{array}{l}21 / 11 \\
96 / 28 \\
15 / 4\end{array}$ & $\begin{array}{l}52.1 \pm 12.4 \\
63.9 \pm 7.0 \\
62.2 \pm 11.8\end{array}$ & $\begin{array}{l}- \\
- \\
-\end{array}$ \\
\hline Yamanaka [3] & 1997 & Japan & $\begin{array}{l}\text { B-HCC } \\
\text { C-HCC } \\
\text { NBNC }\end{array}$ & $\begin{array}{l}27 \\
151 \\
20\end{array}$ & $\begin{array}{l}24 / 3 \\
125 / 26 \\
18 / 2\end{array}$ & $\begin{array}{l}51 \pm 10 \\
63 \pm 6.3 \\
63 \pm 6.4\end{array}$ & $\begin{array}{l}- \\
- \\
-\end{array}$ \\
\hline Wu [26] & 1999 & Taiwan & $\begin{array}{l}\text { B-HCC } \\
\text { C-HCC } \\
\text { NBNC }\end{array}$ & $\begin{array}{l}131 \\
70 \\
40\end{array}$ & $\begin{array}{l}110 / 21 \\
56 / 14 \\
29 / 11\end{array}$ & $\begin{array}{l}54.3 \pm 1.1 \\
64.1 \pm 1.1 \\
68.9 \pm 1.9\end{array}$ & $34.5 * \&$ \\
\hline Shiraishi [32] & 1999 & Japan & $\begin{array}{l}\text { B-HCC } \\
\text { C-HCC } \\
\text { NBNC }\end{array}$ & $\begin{array}{l}11 \\
21 \\
12\end{array}$ & $\begin{array}{l}- \\
- \\
-\end{array}$ & $\begin{array}{l}54.0 \pm 3.2 \\
62.0 \pm 1.8 \\
63.0 \pm 4.1\end{array}$ & $\begin{array}{l}- \\
-\end{array}$ \\
\hline Lee [4] & 2000 & Taiwan & $\begin{array}{l}\text { B-HCC } \\
\text { C-HCC } \\
\text { NBNC }\end{array}$ & $\begin{array}{l}133 \\
66 \\
30\end{array}$ & $\begin{array}{l}112 / 21 \\
48 / 18 \\
20 / 10\end{array}$ & $\begin{array}{l}49.4 \pm 12.7 \\
61.7 \pm 9.2 \\
54.3 \pm 13.3\end{array}$ & $23.5 \pm 16.3 \&$ \\
\hline Noguchi [33] & 2000 & Japan & $\begin{array}{l}\text { B-HCC } \\
\text { C-HCC } \\
\text { NBNC }\end{array}$ & $\begin{array}{l}44 \\
232 \\
13\end{array}$ & $\begin{array}{l}34 / 10 \\
172 / 60 \\
12 / 1\end{array}$ & $\begin{array}{l}51.6 \pm 8.4 \\
65.0 \pm 7.0 \\
60.9 \pm 6.7\end{array}$ & $\begin{array}{l}- \\
- \\
-\end{array}$ \\
\hline Roayaie [34] & 2000 & United States & $\begin{array}{l}\text { B-HCC } \\
\text { C-HCC }\end{array}$ & $\begin{array}{l}21 \\
24\end{array}$ & $\begin{array}{l}10 / 11 \\
17 / 7\end{array}$ & $\begin{array}{l}54.3 \pm 15.3 \\
63.4 \pm 8.5\end{array}$ & $20.3^{*} \&$ \\
\hline Ahmad [35] & 2001 & United States & $\begin{array}{l}\text { B-HCC } \\
\text { C-HCC } \\
\text { NBNC }\end{array}$ & $\begin{array}{l}18 \\
44 \\
15\end{array}$ & $\begin{array}{l}13 / 5 \\
34 / 10 \\
6 / 9\end{array}$ & $\begin{array}{l}60 \\
61 \\
63\end{array}$ & $\begin{array}{l}30^{*} \\
27^{*} \\
33^{*}\end{array}$ \\
\hline Chen [36] & 2001 & Taiwan & $\begin{array}{l}\text { B-HCC } \\
\text { C-HCC }\end{array}$ & $\begin{array}{l}211 \\
59\end{array}$ & $\begin{array}{l}190 / 21 \\
47 / 12\end{array}$ & $\begin{array}{l}57.6 \pm 12.7 \\
66.9 \pm 8.2\end{array}$ & - \\
\hline Wakai [37] & 2003 & Japan & $\begin{array}{l}\text { B-HCC } \\
\text { C-HCC } \\
\text { NBNC }\end{array}$ & $\begin{array}{l}32 \\
55 \\
24\end{array}$ & $\begin{array}{l}20 / 12 \\
46 / 9 \\
18\end{array}$ & $\begin{array}{l}52.5(16-77)^{*} \\
64(46-78) \\
68(45-79)\end{array}$ & $75^{*} \&$ \\
\hline Pawlik [5] & 2004 & Multi center & $\begin{array}{l}\text { B-HCC } \\
\text { C-HCC } \\
\text { NBNC }\end{array}$ & $\begin{array}{l}163 \\
79 \\
126\end{array}$ & $\begin{array}{l}137 / 26 \\
48 / 31 \\
90 / 36\end{array}$ & $\begin{array}{l}60^{*} \\
60 \\
51\end{array}$ & $33^{*} \&$ \\
\hline Uchiyama [39] & 2005 & Japan & $\begin{array}{l}\text { B-HCC } \\
\text { C-HCC } \\
\text { NBNC }\end{array}$ & $\begin{array}{l}25 \\
72 \\
24\end{array}$ & $\begin{array}{l}18 / 7 \\
48 / 24 \\
18 / 6\end{array}$ & $\begin{array}{l}54 \pm 10 \\
64 \pm 9 \\
65 \pm 8\end{array}$ & $\begin{array}{l}- \\
-\end{array}$ \\
\hline Yokoi [38] & 2005 & Japan & $\begin{array}{l}\text { B-HCC } \\
\text { C-HCC } \\
\text { NBNC }\end{array}$ & $\begin{array}{l}25 \\
116 \\
13\end{array}$ & $\begin{array}{l}19 / 6 \\
95 / 21 \\
10 / 3\end{array}$ & $\begin{array}{l}57(32-74)^{*} \\
64(46-85) \\
58(28-72)\end{array}$ & $\begin{array}{l}- \\
- \\
-\end{array}$ \\
\hline Sasaki [40] & 2006 & Japan. & $\begin{array}{l}\text { B-HCC } \\
\text { C-HCC }\end{array}$ & $\begin{array}{l}66 \\
351\end{array}$ & $\begin{array}{l}49 / 17 \\
268 / 83\end{array}$ & $\begin{array}{l}>65(n=5) \\
>65(n=114)\end{array}$ & $\begin{array}{l}132^{*} \\
121.2^{*}\end{array}$ \\
\hline Li [27] & 2007 & China & $\begin{array}{l}\text { B-HCC } \\
\text { C-HCC } \\
\text { NBNC }\end{array}$ & $\begin{array}{l}251 \\
75 \\
54\end{array}$ & $\begin{array}{l}212 / 39 \\
62 / 13 \\
44 / 10\end{array}$ & $\begin{array}{l}51.2 \pm 4.2 \\
63.2 \pm 7.3 \\
67.1 \pm 5.7\end{array}$ & $48.3^{*} \&$ \\
\hline Nanashima [28] & 2007 & Japan & $\begin{array}{l}\text { B-HCC } \\
\text { C-HCC } \\
\text { NBNC }\end{array}$ & $\begin{array}{l}76 \\
124 \\
29\end{array}$ & $\begin{array}{l}61 / 15 \\
99 / 25 \\
21 / 8\end{array}$ & $\begin{array}{l}59 \pm 11 \\
67 \pm 7 \\
65 \pm 8\end{array}$ & $\begin{array}{l}- \\
- \\
-\end{array}$ \\
\hline Kondo [41] & 2008 & Japan & $\begin{array}{l}\text { B-HCC } \\
\text { C-HCC } \\
\text { NBNC }\end{array}$ & $\begin{array}{l}78 \\
127 \\
60\end{array}$ & $\begin{array}{l}58 / 20 \\
94 / 33 \\
43 / 17\end{array}$ & $\begin{array}{l}54.7 \pm 11.6 \\
67.2 \pm 6.7 \\
67.9 \pm 10.3\end{array}$ & $26 * \&$ \\
\hline Cescon [29] & 2009 & Italy & $\begin{array}{l}\text { B-HCC } \\
\text { C-HCC } \\
\text { NBNC }\end{array}$ & $\begin{array}{l}25 \\
130 \\
35\end{array}$ & $\begin{array}{l}24 / 1 \\
90 / 40 \\
30 / 5\end{array}$ & $\begin{array}{l}60.2 \pm 9.8 \\
65.2 \pm 8.1 \\
64.2 \pm 9.1\end{array}$ & $30 * \&$ \\
\hline Kao [42] & 2011 & Taiwan & $\begin{array}{l}\mathrm{B}-\mathrm{HCC} \\
\mathrm{C}-\mathrm{HCC}\end{array}$ & $\begin{array}{l}609 \\
206\end{array}$ & $\begin{array}{l}516 / 93 \\
147 / 59\end{array}$ & $\begin{array}{l}56.3 \pm 13.5 \\
67.2 \pm 9.1\end{array}$ & $40.6^{*} \&$ \\
\hline
\end{tabular}


Table 2 Results of a meta-analysis

\begin{tabular}{|c|c|c|c|c|c|c|c|}
\hline Outcome of interest & No. of studies & $\begin{array}{l}\text { No.of } \\
\text { patients }\end{array}$ & Results & OR/WMD & $95 \% \mathrm{Cl}$ & $P$-value & $\mathrm{I}^{2}(\%)$ \\
\hline \multicolumn{8}{|l|}{ Patients characteristics } \\
\hline \multicolumn{8}{|l|}{ Age (years) } \\
\hline $\mathrm{B}-\mathrm{HCC}$ versus $\mathrm{C}-\mathrm{HCC}$ & $15[3,4,26-34,36,39,41,42]$ & 3281 & $\mathrm{~B}-\mathrm{HCC}=54.4 \pm 9.2, \mathrm{C}-\mathrm{HCC}=64.3 \pm 6.8$ & -10.11 & $-11.14,-9.09$ & $<0.001$ & 65.3 \\
\hline B-HCC versus NBNC-HCC & $11[3,4,26,27,29,31-33,39,41]$ & 1169 & $\mathrm{~B}-\mathrm{HCC}=53.7 \pm 8.5, \mathrm{NBNC}-\mathrm{HCC}=63.7 \pm 7.7$ & -10.42 & $-12.72,-8.12$ & $<0.001$ & 86.1 \\
\hline $\mathrm{C}-\mathrm{HCC}$ versus NBNC-HCC & $11[3,4,26,27,29,31-33,39,41]$ & 1528 & $\mathrm{C}-\mathrm{HCC}=64.2 \pm 6.4, \mathrm{NBNC}-\mathrm{HCC}=63.7 \pm 7.7$ & 0.08 & $-2.18,2.38$ & 0.95 & 88.2 \\
\hline \multicolumn{8}{|l|}{ Male } \\
\hline $\mathrm{B}-\mathrm{HCC}$ versus $\mathrm{C}-\mathrm{HCC}$ & $19[3-5,26-31,33-42]$ & 4198 & $\mathrm{~B}-\mathrm{HCC}=82.6 \%, \mathrm{C}-\mathrm{HCC}=75.8 \%$ & 1.19 & $0.89,1.60$ & 0.24 & 61.2 \\
\hline $\mathrm{B}-\mathrm{HCC}$ versus NBNC-HCC & $14[3-5,26-29,31,33,35,37-39,41]$ & 1562 & $\mathrm{~B}-\mathrm{HCC}=81.4 \%, \mathrm{NBNC}-\mathrm{HCC}=74.5 \%$ & 1.43 & $1.10,1.86$ & 0.008 & 16.3 \\
\hline $\mathrm{C}-\mathrm{HCC}$ versus NBNC-HCC & $14[3-5,26-29,31,33,35,37-39,41]$ & 1967 & $\mathrm{C}-\mathrm{HCC}=75.9 \%, \mathrm{NBNC}-\mathrm{HCC}=74.5 \%$ & 0.96 & $0.74,1.23$ & 0.74 & 31 \\
\hline \multicolumn{8}{|l|}{ Liver function } \\
\hline \multicolumn{8}{|l|}{ Serum ALT level (IU/I) } \\
\hline $\mathrm{B}-\mathrm{HCC}$ versus $\mathrm{C}-\mathrm{HCC}$ & $11[3,4,27,29,30,32-34,36,39,41]$ & 1909 & $\mathrm{~B}-\mathrm{HCC}=56.4 \pm 44.8, \mathrm{C}-\mathrm{HCC}=76.9 \pm 47.6$ & -16.84 & $-21.02,-12.65$ & $<0.001$ & 23.4 \\
\hline B-HCC versus NBNC-HCC & $8[3,4,27,29,32,33,39,41]$ & 842 & $\mathrm{~B}-\mathrm{HCC}=56.7 \pm 55.9, \mathrm{NBNC}-\mathrm{HCC}=39.6 \pm 31.1$ & 15.30 & $4.59,26.01$ & 0.005 & 73.9 \\
\hline $\mathrm{C}-\mathrm{HCC}$ versus NBNC-HCC & $8[3,4,27,29,32,33,39,41]$ & 1122 & $\mathrm{C}-\mathrm{HCC}=74.1 \pm 43.8, \mathrm{NBNC}-\mathrm{HCC}=39.6 \pm 31.1$ & 34.41 & $23.75,45.08$ & $<0.001$ & 84.9 \\
\hline \multicolumn{8}{|l|}{ Serum AST level (IU/I) } \\
\hline $\mathrm{B}-\mathrm{HCC}$ versus $\mathrm{C}-\mathrm{HCC}$ & $8[3,4,29,30,32,33,39,41]$ & 842 & $\mathrm{~B}-\mathrm{HCC}=60.0 \pm 56.7, \mathrm{C}-\mathrm{HCC}=70.8 \pm 38.1$ & -13.17 & $-22.29,-4.05$ & 0.005 & 61.5 \\
\hline B-HCC versus NBNC-HCC & $7[3,4,29,32,33,39,41]$ & 537 & $\mathrm{~B}-\mathrm{HCC}=61.0 \pm 55.9, \mathrm{NBNC}-\mathrm{HCC}=43.8 \pm 25.5$ & 13.06 & $0.13,26.00$ & 0.05 & 72.8 \\
\hline $\mathrm{C}-\mathrm{HCC}$ versus NBNC-HCC & $7[3,4,29,32,33,39,41]$ & 993 & $\mathrm{C}-\mathrm{HCC}=69.9 \pm 37.7, \mathrm{NBNC}-\mathrm{HCC}=43.8 \pm 25.5$ & 24.87 & $18.94,30.79$ & $<0.001$ & 56.5 \\
\hline \multicolumn{8}{|l|}{ Serum albumin level (g/dl) } \\
\hline $\mathrm{B}-\mathrm{HCC}$ versus $\mathrm{C}-\mathrm{HCC}$ & $10[3,27,29-31,33,34,36,39,41]$ & 1834 & $\mathrm{~B}-\mathrm{HCC}=3.93 \pm 0.48, \mathrm{C}-\mathrm{HCC}=3.69 \pm 0.48$ & 0.23 & $0.08,0.38$ & 0.002 & 87.4 \\
\hline B-HCC versus NBNC-HCC & $7[3,27,29,31,33,39,41]$ & 707 & $\mathrm{~B}-\mathrm{HCC}=3.91 \pm 0.45, \mathrm{NBNC}-\mathrm{HCC}=3.94 \pm 0.48$ & -0.07 & $-0.15,0.00$ & 0.07 & 36.4 \\
\hline $\mathrm{C}-\mathrm{HCC}$ versus NBNC-HCC & $7[3,27,29,31,33,39,41]$ & 1136 & $\mathrm{C}-\mathrm{HCC}=3.61 \pm 0.47, \mathrm{NBNC}-\mathrm{HCC}=3.94 \pm 0.48$ & -0.29 & $-0.53,-0.05$ & 0.002 & 89.7 \\
\hline \multicolumn{8}{|l|}{ ICG R15 (\%) } \\
\hline $\mathrm{B}-\mathrm{HCC}$ versus $\mathrm{C}-\mathrm{HCC}$ & $10[3,4,26,31-33,36,39,41]$ & 1740 & $\mathrm{~B}-\mathrm{HCC}=12.9 \pm 7.8, \mathrm{C}-\mathrm{HCC}=20.4 \pm 9.1$ & -6.58 & $-8.3,-4.87$ & $<0.001$ & 78.9 \\
\hline B-HCC versus NBNC-HCC & $8[3,4,26,31-33,39,41]$ & 699 & $\mathrm{~B}-\mathrm{HCC}=12.8 \pm 7.5, \mathrm{NBNC}-\mathrm{HCC}=13.9 \pm 7.7$ & -0.74 & $-1.77,-0.30$ & 0.16 & 21.3 \\
\hline $\mathrm{C}-\mathrm{HCC}$ versus NBNC-HCC & $8[3,4,26,31-33,39,41]$ & 1081 & $\mathrm{C}-\mathrm{HCC}=21.0 \pm 9.0, \mathrm{NBNC}-\mathrm{HCC}=13.9 \pm 7.7$ & 5.92 & $3.85,7.99$ & $<0.001$ & 74.3 \\
\hline \multicolumn{8}{|l|}{ Child's grade A } \\
\hline $\mathrm{B}-\mathrm{HCC}$ versus $\mathrm{C}-\mathrm{HCC}$ & $9[4,5,27,28,32,35,38,40,42]$ & 2434 & $\mathrm{~B}-\mathrm{HCC}=88.3 \%, \mathrm{C}-\mathrm{HCC}=80.8 \%$ & 1.68 & $1.25,2.25$ & $<0.001$ & 34.9 \\
\hline B-HCC versus NBNC-HCC & $7[4,5,27,28,32,35,38]$ & 956 & B-HCC $=79.4 \%$, NBNC-HCC $=80.6 \%$ & 1.31 & $0.87,1.98$ & 0.20 & 0 \\
\hline $\mathrm{C}-\mathrm{HCC}$ versus NBNC-HCC & $7[4,5,27,28,32,35,38]$ & 804 & $\mathrm{C}-\mathrm{HCC}=78.4 \%, \mathrm{NBNC}-\mathrm{HCC}=80.6 \%$ & 0.69 & $0.46,1.05$ & 0.08 & 1.1 \\
\hline \multicolumn{8}{|l|}{ Serum T-Bil level (mg/dL) } \\
\hline $\mathrm{B}-\mathrm{HCC}$ versus $\mathrm{C}-\mathrm{HCC}$ & $9[4,27,29-31,33,36,39,41]$ & 1579 & $\mathrm{~B}-\mathrm{HCC}=0.91 \pm 0.47, \mathrm{C}-\mathrm{HCC}=1.23 \pm 0.83$ & -0.14 & $-0.27,-0.01$ & 0.03 & 80.1 \\
\hline B-HCC versus NBNC-HCC & $6[4,27,29,31,39,41]$ & 766 & $\mathrm{~B}-\mathrm{HCC}=0.92 \pm 0.47, \mathrm{NBNC}-\mathrm{HCC}=0.87 \pm 0.49$ & 0.06 & $-0.16,0.28$ & 0.60 & 90.3 \\
\hline $\mathrm{C}-\mathrm{HCC}$ versus NBNC-HCC & $6[4,27,29,31,39,41]$ & 816 & $\mathrm{C}-\mathrm{HCC}=1.18 \pm 0.76, \mathrm{NBNC}-\mathrm{HCC}=0.87 \pm 0.49$ & 0.25 & $-0.02,0.52$ & 0.07 & 90.6 \\
\hline \multicolumn{8}{|l|}{ Serum platelet count $\left(\times 10^{3} / \mathrm{mm}\right)$} \\
\hline $\mathrm{B}-\mathrm{HCC}$ versus $\mathrm{C}-\mathrm{HCC}$ & $7[27,29,30,33,34,39,41]$ & 1230 & $\mathrm{~B}-\mathrm{HCC}=166.6 \pm 85.0, \mathrm{C}-\mathrm{HCC}=137.5 \pm 66.9$ & 24.47 & $1.24,47.7$ & 0.04 & 82.1 \\
\hline B-HCC versus NBNC-HCC & $5[27,29,33,39,41]$ & 609 & $\mathrm{~B}-\mathrm{HCC}=156.8 \pm 73.4, \mathrm{NBNC}-\mathrm{HCC}=192.2 \pm 72.4$ & -28.88 & $-41.93,-15.83$ & $<0.001$ & 30.9 \\
\hline
\end{tabular}


Table 2 Results of a meta-analysis (Continued)

\begin{tabular}{|c|c|c|c|c|c|c|c|}
\hline $\mathrm{C}-\mathrm{HCC}$ versus NBNC-HCC & $5[27,29,33,39,41]$ & 822 & $\mathrm{C}-\mathrm{HCC}=138.2 \pm 66.6, \mathrm{NBNC}-\mathrm{HCC}=192.2 \pm 72.4$ & -50.43 & $-75.13,-25.72$ & $<0.001$ & 74.8 \\
\hline \multicolumn{8}{|l|}{ Tumor characteristics } \\
\hline \multicolumn{8}{|l|}{ Size $(\mathrm{cm})$} \\
\hline $\mathrm{B}-\mathrm{HCC}$ versus $\mathrm{C}-\mathrm{HCC}$ & $10[3,26,27,29,30,33,34,36,39,41]$ & 1879 & $\mathrm{~B}-\mathrm{HCC}=5.4 \pm 2.5, \mathrm{C}-\mathrm{HCC}=4.0 \pm 2.1$ & 1.32 & $0.38,2.27$ & 0.006 & 98.4 \\
\hline B-HCC versus NBNC-HCC & $7[3,26,27,29,33,39,41]$ & 827 & $\mathrm{~B}-\mathrm{HCC}=5.1 \pm 2.5, \mathrm{NBNC}-\mathrm{HCC}=5.3 \pm 2.6$ & -0.02 & $-0.94,0.00$ & 0.97 & 96.5 \\
\hline $\mathrm{C}-\mathrm{HCC}$ versus NBNC-HCC & $7[3,26,27,29,33,39,41]$ & 1103 & $\mathrm{C}-\mathrm{HCC}=3.8 \pm 2.2, \mathrm{NBNC}-\mathrm{HCC}=5.3 \pm 2.6$ & -0.86 & $-1.27,-0.45$ & $<0.001$ & 78.1 \\
\hline \multicolumn{8}{|l|}{ Coexisting cirrhosis } \\
\hline $\mathrm{B}-\mathrm{HCC}$ versus $\mathrm{C}-\mathrm{HCC}$ & $15[3,4,26-32,34-38,40-42]$ & 3623 & $\mathrm{~B}-\mathrm{HCC}=53.4 \%, \mathrm{C}-\mathrm{HCC}=65.7 \%$ & 0.71 & $0.54,0.92$ & 0.01 & 55.5 \\
\hline B-HCC versus NBNC-HCC & $12[3,4,26-29,31,32,35,37,38,41]$ & 1190 & $\mathrm{~B}-\mathrm{HCC}=61.8 \%, \mathrm{NBNC}-\mathrm{HCC}=45.5 \%$ & 2.61 & $1.56,4.64$ & $<0.001$ & 63.1 \\
\hline $\mathrm{C}-\mathrm{HCC}$ versus NBNC-HCC & $12[3,4,26-29,31,32,35,37,38,41]$ & 1454 & $\mathrm{C}-\mathrm{HCC}=69.9 \%, \mathrm{NBNC}-\mathrm{HCC}=45.5 \%$ & 3.92 & $2.35,6.53$ & $<0.001$ & 56.4 \\
\hline \multicolumn{8}{|l|}{ Vascular invasion } \\
\hline B-HCC versus C-HCC & $17[3-5,26-30,34-42]$ & 3760 & $\mathrm{~B}-\mathrm{HCC}=46.2 \%, \mathrm{C}-\mathrm{HCC}=34.4 \%$ & 1.29 & $0.97,1.73$ & 0.08 & 61.2 \\
\hline B-HCC versus NBNC-HCC & $12[3-5,26-29,35,37-39,42]$ & 1454 & $\mathrm{~B}-\mathrm{HCC}=31.9 \%, \mathrm{NBNC}-\mathrm{HCC}=32.9 \%$ & 1.44 & $0.99,2.11$ & 0.06 & 37.5 \\
\hline C-HCC versus NBNC-HCC & $12[3-5,26-29,35,37-39,42]$ & 1579 & $\mathrm{C}-\mathrm{HCC}=28.7 \%, \mathrm{NBNC}-\mathrm{HCC}=32.9 \%$ & 0.99 & $0.62,1.56$ & 0.96 & 59.0 \\
\hline \multicolumn{8}{|c|}{ Intrahepatic metastases/satellite nodules } \\
\hline $\mathrm{B}-\mathrm{HCC}$ versus $\mathrm{C}-\mathrm{HCC}$ & $11[4,26,28-31,35,37-40]$ & 1836 & $\mathrm{~B}-\mathrm{HCC}=31 \%, \mathrm{C}-\mathrm{HCC}=24.5 \%$ & 1.23 & $0.87,1.73$ & 0.24 & 42.0 \\
\hline B-HCC versus NBNC-HCC & $9[4,26,28,29,31,35,37-39]$ & 726 & $\mathrm{~B}-\mathrm{HCC}=30.3 \%, \mathrm{NBNC}-\mathrm{HCC}=28.8 \%$ & 1.01 & $0.56,1.83$ & 0.97 & 49.9 \\
\hline $\mathrm{C}-\mathrm{HCC}$ versus NBNC-HCC & $9[4,26,28,29,31,35,37-39]$ & 1030 & $\mathrm{C}-\mathrm{HCC}=24.4 \%, \mathrm{NBNC}-\mathrm{HCC}=28.8 \%$ & 0.98 & $0.69,1.39$ & 0.91 & 23.8 \\
\hline \multicolumn{8}{|l|}{ Capsule formation } \\
\hline B-HCC versus C-HCC & $8[4,26,27,29,30,36,38,39]$ & 1509 & $\mathrm{~B}-\mathrm{HCC}=47.7 \%, \mathrm{C}-\mathrm{HCC}=53.8 \%$ & 0.86 & $0.57,1.29$ & 0.46 & 53.8 \\
\hline B-HCC versus NBNC-HCC & $6[4,26,27,29,38,39]$ & 786 & $\mathrm{~B}-\mathrm{HCC}=49.3 \%, \mathrm{NBNC}-\mathrm{HCC}=47.9 \%$ & 0.96 & $0.55,1.67$ & 0.88 & 51.1 \\
\hline $\mathrm{C}-\mathrm{HCC}$ versus NBNC-HCC & $6[4,26,27,29,38,39]$ & 725 & $\mathrm{C}-\mathrm{HCC}=52.1 \%, \mathrm{NBNC}-\mathrm{HCC}=47.9 \%$ & 1.10 & $0.77,1.57$ & 0.60 & 18.9 \\
\hline \multicolumn{8}{|l|}{ Serum AFP level (ng/ml) } \\
\hline $\mathrm{B}-\mathrm{HCC}$ versus $\mathrm{C}-\mathrm{HCC}$ & $9[3,28-31,33,34,36,41]$ & 1611 & B-HCC $=11555.3 \pm 45653.8, \mathrm{C}-\mathrm{HCC}=2496.0 \pm 9014.5$ & -52.96 & $-281.61,175.69$ & 0.65 & 39.5 \\
\hline B-HCC versus NBNC-HCC & $6[3,28,29,31,33,41]$ & 458 & B-HCC $=13927.5 \pm 56323.0$, NBNC-HCC $=3069.1 \pm 9330.6$ & 1385.80 & $-1099.05,3870.66$ & 0.27 & 86.8 \\
\hline C-HCC versus NBNC-HCC & $6[3,28,29,31,33,41]$ & 1064 & $\mathrm{C}-\mathrm{HCC}=2181.0 \pm 8052.8, \mathrm{NBNC}-\mathrm{HCC}=3069.1 \pm 9330.6$ & -214.61 & $-714.20,284.98$ & 0.40 & 0 \\
\hline \multicolumn{8}{|l|}{ Survival } \\
\hline \multicolumn{8}{|l|}{ 5-year overall survival } \\
\hline $\mathrm{B}-\mathrm{HCC}$ versus $\mathrm{C}-\mathrm{HCC}$ & $14[3-5,26-28,30,34-36,38,40-42]$ & 3427 & $\mathrm{~B}-\mathrm{HCC}=51.4 \%, \mathrm{C}-\mathrm{HCC}=52.9 \%$ & 1.00 & $0.76,1.31$ & 0.99 & 61.9 \\
\hline B-HCC versus NBNC-HCC & $9[3-5,26-28,35,38,41]$ & 1289 & $\mathrm{~B}-\mathrm{HCC}=50.2 \%, \mathrm{NBNC}-\mathrm{HCC}=53.0 \%$ & 0.68 & $0.44,1.06$ & 0.09 & 55.8 \\
\hline $\mathrm{C}-\mathrm{HCC}$ versus NBNC-HCC & $9[3-5,26-28,35,38,41]$ & 1239 & $\mathrm{C}-\mathrm{HCC}=49.0 \%, \mathrm{NBNC}-\mathrm{HCC}=53.0 \%$ & 0.61 & $0.33,1.11$ & 0.10 & 75.7 \\
\hline \multicolumn{8}{|l|}{ 5-year disease-free survival } \\
\hline $\mathrm{B}-\mathrm{HCC}$ versus $\mathrm{C}-\mathrm{HCC}$ & $13[3,4,26,28-30,34,35,37-41]$ & 2113 & $\mathrm{~B}-\mathrm{HCC}=32.3 \%, \mathrm{C}-\mathrm{HCC}=25.5 \%$ & 1.46 & $0.88,2.41$ & 0.14 & 77.8 \\
\hline B-HCC versus NBNC-HCC & $10[3,4,26,28,29,35,37-39,41]$ & 860 & $\mathrm{~B}-\mathrm{HCC}=28.7 \%, \mathrm{NBNC}-\mathrm{HCC}=49.3 \%$ & 0.39 & $0.28,0.53$ & $<0.001$ & 33.4 \\
\hline $\mathrm{C}-\mathrm{HCC}$ versus NBNC-HCC & $10[3,4,26,28,29,35,37-39,41]$ & 1245 & $\mathrm{C}-\mathrm{HCC}=26.8 \%, \mathrm{NBNC}-\mathrm{HCC}=49.3 \%$ & 0.37 & $0.22,0.64$ & $<0.001$ & 69.4 \\
\hline
\end{tabular}

OR = odds ratio; WMD = weighted mean difference; $\mathrm{Cl}=$ confidence interval, $\mathrm{HCC}=$ hepatocellular carcinoma; B-HCC = hepatitis B-related hepatocellular carcinoma; $\mathrm{C}-\mathrm{HCC}=$ hepatitis C-related hepatocellular indocyanine green retention rate at 15 minutes. 
to $-8.12, P<0.001)$. The prevalence of male sex was higher in the B-HCC group than in the NBNC-HCC group (OR: $1.43,95 \%$ CI: 1.10 to $1.86, P=0.008$ ). They also were more male in the B-HCC group than in the $\mathrm{C}$-HCC group, although the differences were not statistically significant $(P=0.24)$.

\section{Liver Function}

Serum aspartate aminotransferase and alanine aminotransferase levels were higher in the C-HCC group than in the other two groups. The serum total bilirubin level and indocyanine green retention rate at $15 \mathrm{~min}$ were higher, and the serum albumin level was lower in the $\mathrm{C}$ $\mathrm{HCC}$ group than in the NBNC-HCC group. The platelet count was higher in the NBNC-HCC group than in the other two groups. The Child's grade A was more frequently recognized in the B-HCC group than in the CHCC group (Table 2).

\section{Tumor characteristics}

The mean tumor size was significantly larger in B-HCC and NBNC-HCC group than in $\mathrm{C}-\mathrm{HCC}$ group (respectively: WMD: 1.32 , 95\% CI: 0.38 to $2.27, P=0.006$; WMD: $-0.86,95 \% \mathrm{CI}:-1.27$ to $-0.45, P<0.001)$. No significant differences were observed between B-HCC and NBNC-HCC group but NBNC-HCC group tended to have larger tumors $(P=0.97)$. The prevalence of liver cirrhosis was the highest in the $\mathrm{C}-\mathrm{HCC}$ group, followed by the B-HCC group, and the NBNC-HCC group $(P<$ 0.01 ). The incidence of vascular invasion, intrahepatic metastases/satellite nodules, tumor capsule formation, and serum AFP level, all were similar in the three groups (Table 2).

\section{Survival}

There was a tendency toward higher 5-year overall survival rates in the NBNC-HCC group compared to those in the other two groups, although these differences were not statistically significant (Table 2).

Pooled analysis of studies furnishing data found that patients with HBV or HCV infection had a worse 5-year disease-free survival when compared to patients with NBNC-HCC (respectively: OR: 0.39, 95\% CI: 0.28 to $0.53, P<0.001$; WMD: $0.37,95 \%$ CI: 0.22 to $0.64, P<$ 0.001 ) (Figure 1, 2 and 3).

Both the 5-year overall survival and disease-free survival in the B-HCC and C-HCC groups were not significantly different (Table 2 ).

\section{Discussion}

HBV belongs to a family of DNA viruses called hepadnaviruses. The oncogenic potential of HBV has been attributed to its ability to integrate into host cellular DNA, which, may activate neighboring cellular genes directly to offer a selective growth advantage to the liver cells. In addition, production of hepatitis $B \times(\mathrm{HBx})$ protein can act as a transactivator on various cellular genes for cell growth and tumorigenesis [43]. In contrast, $\mathrm{HCV}$ is a positive-stranded RNA virus the genome of which does not seem to integrate into hepatocyte's genome [44]. Therefore, differences in carcinogenetic mechanisms between these viruses may affect HCC characteristics.

Most chronic HBV infections are vertical transmissions during delivery, whereas $\mathrm{HCV}$ infections are known to be blood-borne such as from transfusions and occurs mainly after the age of 20 years. Consequently,

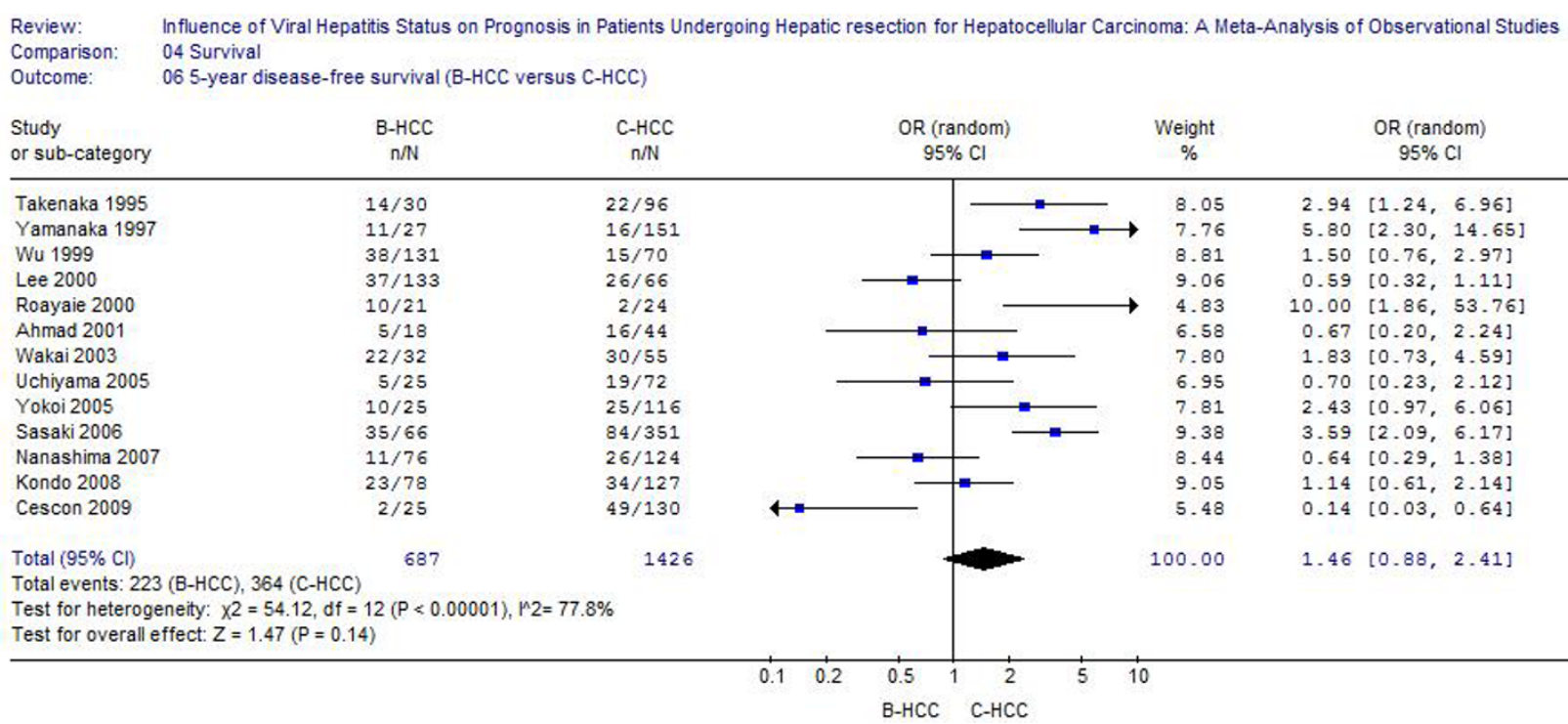

Figure 1 B-HCC versus C-HCC: Results of the meta-analysis on 5-year disease-free survival. All based on a random-effects meta-analysis. 


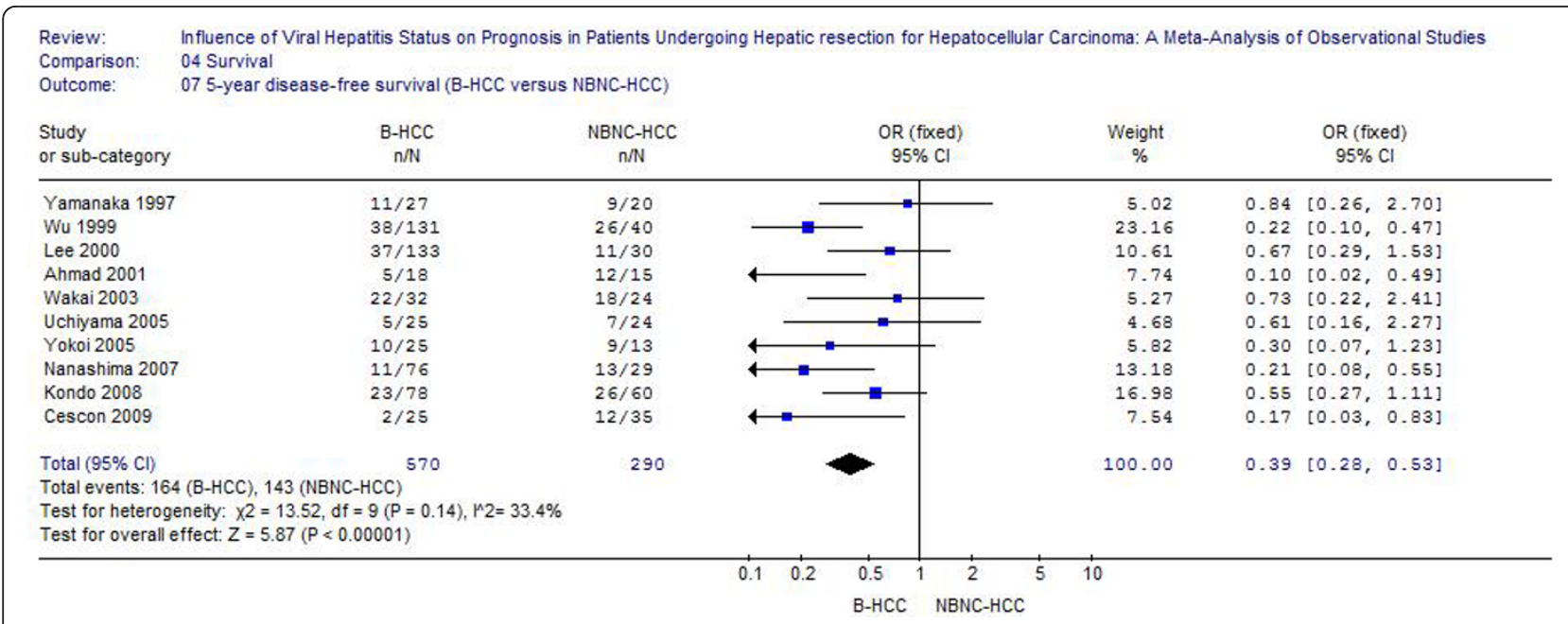

Figure 2 B-HCC versus NBNC-HCC: Results of the meta-analysis on 5-year disease-free survival. All based on a fixed-effects meta-analysis.

the mean age at occurrence of $\mathrm{HCC}$ is lower in $\mathrm{B}-\mathrm{HCC}$ than in $\mathrm{C}$-HCC. Interestingly, we also found that the mean age for patients with NBNC-HCC is significantly older than the B-HCC group. It is suspected that NBNC-HCC requires a longer time until it develops HCC [33]. The liver cirrhosis was more frequently recognized in the $\mathrm{C}-\mathrm{HCC}$ group than in the $\mathrm{B}-\mathrm{HCC}$ and NBNC-HCC groups. Thus, as reflected by many parameters, among the three groups, liver function was the worst in the $\mathrm{C}-\mathrm{HCC}$ group.

$\mathrm{HCC}$ is more prevalent in men than in women, this trend is less apparent for patients with HCC unrelated to HBV. Both animal and human studies support the importance of androgen signaling in determining the male preference of HCC [45]. Increased expression and activation of androgen receptor (AR) was found in HCC and nontumorous liver tissue [46]. A recent study demonstrated that the HBx protein increased the anchorage-independent colony-formation potency of AR in a nontransformed mouse hepatocyte cell line. In addition, $\mathrm{HBx}$ functioned as a positive transcriptional coregulator to increase AR-mediated transcriptional activity [47]. These findings may provide a plausible explanation for the male gender preference of HBV-related HCC.

With respect to tumor factors, this study demonstrated that patients in the NBNC-HCC group had largest tumors. This was probably due to fewer NBNC-HCC patients receiving regular follow-up for the liver diseases since the two major risk factors for $\mathrm{HCC}, \mathrm{HBV}$ and $\mathrm{HCV}$, were negative $[6,7,33]$. The HCC might be

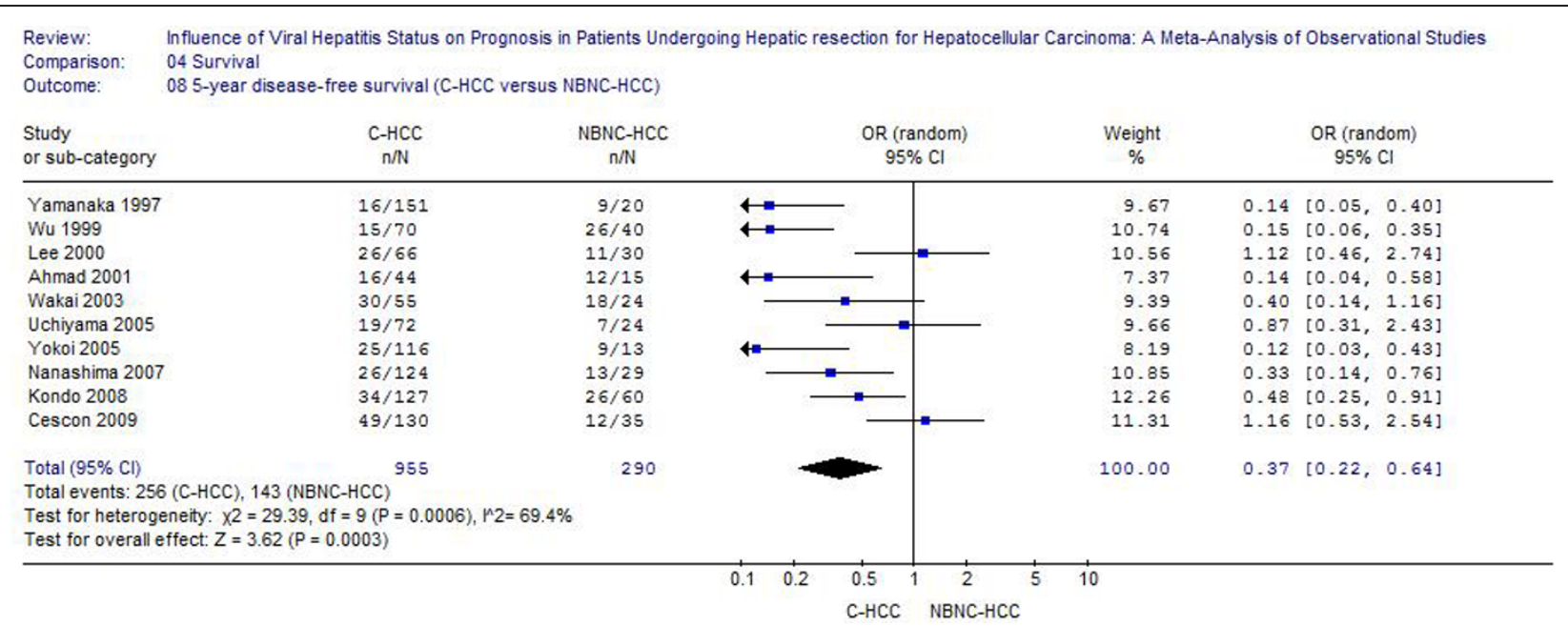

Figure 3 C-HCC versus NBNC-HCC: Results of the meta-analysis on 5-year disease-free survival. All based on a random-effects metaanalysis. 
discovered only when the tumor increases in size and caused subjective symptoms in the NBNC-HCC patients. The smaller tumors in the $\mathrm{C}-\mathrm{HCC}$ group may be explained by the fact that $\mathrm{C}-\mathrm{HCC}$ occurring at a much older age. Older age with possible comorbidities and relatively poor liver function usually preclude $\mathrm{C}$-HCC patients with larger tumors from undergoing surgery [42].

In the present study, 5-year disease-free survival rates were significantly higher in the NBNC-HCC group than in the B-HCC and C-HCC groups. High rate of intrhepatic recurrence after surgical resection is the main cause of late death of patients with HCC [48]. According to point of recurrences time from the date of hepatectomy, recurrences were classified into early ( $\leq 2$ year) and late (>2 year) recurrences [49]. Early recurrences appear to arise mainly from intrahepatic metastases from residues of original $\mathrm{HCC}$, whereas late recurrences are more likely to develop on the basis of underlying liver diseases, resulting from new carcinogenesis. It is generally accepted that virus-induced chronic inflammatory necrosis and hepatocyte necrosis might cause the hepatocytes to undergo proliferation and thus increase the occurrence of genetic aberrations, which may be the main mechanism responsible for late intrahepatic recurrence [49]. Wakai et al. [37] found that the cumulative probability of intrahepatic recurrence reached a plateau at 2.4 years after resection in the NBNC group, while it continued to increase steadily in the hepatitis viral groups. Thus, improved disease-free survival in the NBNC-HCC group is attributed to a low incidence of multicentric carcinogenesis, which is caused by chronic viral attack. In addition, NBNC patients maintained good liver function following the initial hepatectomy, and these biological advantages provided NBNC patients more opportunities for repeat resection of intrahepatic recurrences, which may lead to a favorable outcome [38].

Both the 5-year overall survival and disease-free survival in the B-HCC and C-HCC groups were not significantly different, indicating that influence of the viral etiology on the outcome of resection surgery in HCC patients was not obvious.

As a limitation, there are important heterogeneities between studies. There are many differences between the studies that serve as sources of heterogeneity, including variation in surgical skill, variation in perioperative and postoperative care. The other main source to the heterogeneity is NBNC-HCC group and the $\mathrm{C}-\mathrm{HCC}$ group may have included patients with HBV. It was demonstrated that HBV DNA can be detected in the hepatic parenchyma of many HBsAg-negative HCC patients [50]. However, the determination of HBV DNA in liver tissue is not routinely checked during the clinical course of HCC. Given this heterogeneity, we applied a random effect model to take between study variation into consideration. This does not necessarily rule out the effect of heterogeneity between studies, but one may expect a very limited influence. Another limitation is all of data in the present study comes from observational studies. Observational studies are subject to a number of biases, including recall and selection [51]. In addition, since HCC is found commonly in China and other parts of South East Asia, most studies included in current meta-analysis were performed in Asian patients and the data cannot be extrapolated to the non- Asian population.

\section{Conclusions}

Our meta-analysis showed HCC patients with viral infection had a poor prognosis compared to patients with negative serology. It is hypothesized that antiviral therapies would help prevent $\mathrm{HCC}$ recurrence by cleaning the carcinogenic soil and eliminating possibilities of novel tumorigenesis through their viral suppression and antiinflammation action. This theory is supported by a recently published meta-analysis, in that study postoperative adjuvant antiviral therapy has a significant beneficial effect after curative treatment of HBV/HCV related HCC in terms of both survival and tumor recurrence [52]. Thus, for HCC patients with viral infections, postoperative adjuvant antiviral therapy is needed to improve the outcome.

\section{Author details}

'Department of Hepato-Biliary-Pancreato-Vascular Surgery, the First affiliated Hospital of Xiamen University, Xiamen, China. ${ }^{2}$ Department of Blood Transfution, the First affiliated Hospital of Xiamen University, Xiamen, China. ${ }^{3}$ Cancer Center, the First affiliated Hospital of Xiamen University, Xiamen, China.

\section{Authors' contributions}

YZ participated in the design and coordination of the study, carried out the critical appraisal of studies and wrote the manuscript. LW, XS, and XS developed the literature search, carried out the extraction of data, assisted in the critical appraisal of included studies and assisted in writing up. YZ, ZZ, and BL carried out the statistical analysis of studies. All authors read and approved the final manuscript.

\section{Competing interests}

The authors declare that they have no competing interests.

Received: 9 July 2011 Accepted: 21 September 2011

Published: 21 September 2011

\section{References}

1. Llovet JM, Burroughs A, Bruix J: Hepatocellular carcinoma. Lancet 2003, 362:1907-17

2. Bosch FX, Ribes J, Díaz M, Cléries R: Primary liver cancer: worldwide incidence and trends. Gastroenterology 2004, 127:S5-S16.

3. Yamanaka N, Tanaka T, Tanaka W, Yamanaka J, Yasui C, Kuroda N, Takada M, Okamoto E: Correlation of hepatitis virus serologic status with clinicopathologic features in patients undergoing hepatectomy for hepatocellular carcinoma. Cancer 1997, 79:1509-15.

4. Lee WC, Jeng LB, Chen MF: Hepatectomy for hepatitis B-, hepatitis C-, and dual hepatitis B- and C-related hepatocellular carcinoma in Taiwan. $J$ Hepatobiliary Pancreat Surg 2000, 73:265-9. 
5. Pawlik TM, Poon RT, Abdalla EK, Sarmiento JM, Ikai I, Curley SA, Nagorney DM, Belghiti J, Ng IO, Yamaoka Y, Lauwers GY, Vauthey JN: Hepatitis serology predicts tumor and liver-disease characteristics but not prognosis after resection of hepatocellular carcinoma. J Gastrointest Surg 2004, 8:794-804

6. Huo TI, Huang YH, Hsia CY, Su CW, Lin HC, Hsu CY, Lee PC, Lui WY, Loong CC, Chiang JH, Chiou YY, Lee SD: Characteristics and outcome of patients with dual hepatitis $B$ and $C$-associated hepatocellular carcinoma: are they different from patients with single virus infection? Liver Int 2009, 29:767-73

7. Akahoshi $\mathrm{H}$, Taura $\mathrm{N}$, Ichikawa T, Miyaaki H, Akiyama M, Miuma S, Ozawa E, Takeshita S, Muraoka T, Matsuzaki T, Ohtani M, Isomoto H, Matsumoto T, Takeshima F, Nakao K: Differences in prognostic factors according to viral status in patients with hepatocellular carcinoma. Oncol Rep 2010, 23:1317-23.

8. Aziz O, Constantinides V, Tekkis PP, Athanasiou T, Purkayastha S, Paraskeva P, Darzi AW, Heriot AG: Laparoscopic versus open surgery for rectal cancer: a meta-analysis. Ann Surg Oncol 2006, 13:413-24.

9. Rao B, Han M, Wang L, Gao X, Huang J, Huang M, Liu H, Wang J: Clinical outcomes of active specific immunotherapy in advanced colorectal cancer and suspected minimal residual colorectal cancer: a metaanalysis and system review. J Trans/ Med 2011, 9:17.

10. Shiratori $Y$, Shiina S, Imamura M, Kato N, Kanai F, Okudaira T, Teratani T, Tohgo G, Toda N, Ohashi M, et al: Characteristic difference of hepatocellular carcinoma between hepatitis B- and C- viral infection in Japan. Hepatology 1995, 22:1027-33.

11. Tanizaki H, Ryu M, Kinoshita T, Kawano N, Konishi M, Cho A, Nakatsura T, Natsume T, Takahashi S, Sugita M, Izuishi K, Yoshino M, Furuse J, Iwasaki M, Tsubono $Y$ : Comparison of clinical features and survival in patients with hepatitis B and C virus-related hepatocellular carcinoma. Jpn J Clin Oncol 1997, 27:67-70.

12. Tanabe G, Nuruki K, Baba Y, Imamura Y, Miyazono N, Ueno K, Arima T, Nakajyou M, Aikou T: A comparison of hepatocellular carcinoma associated with HBV or HCV infection. Hepatogastroenterology 1999, $46: 2442-6$

13. Watabe $H$, Shiratori $Y$, Tateishi $R$, Fujishima $T$, Akamatsu M, Koike $Y$, Obi $S$, Hamamura K, Sato S, Teratani T, Shiina S, Omata M: Clinical features of patients with HCC who are negative for both HBV and HCV markers. Hepatogastroenterology 2003, 50:2157-60.

14. Dohmen $\mathrm{K}$, Shigematsu $H$, Irie $K$, Ishibashi $H$ : Comparison of the clinical characteristics among hepatocellular carcinoma of hepatitis $B$, hepatitis $C$ and non-B non-C patients. Hepatogastroenterology 2003 50:2022-7.

15. Tangkijvanich $P$, Suwangool $P$, Mahachai $V$ : Comparison of clinical features and survival of patients with hepatitis B- and hepatitis C-associated hepatocellular carcinoma in Thailand. J Med Assoc Thai 2003, 86(Suppl 2): S250-6.

16. Koike $Y$, Shiratori $Y$, Sato $S$, Obi S, Teratani T, Imamura M, Hamamura K, Imai $Y$, Yoshida $H$, Shiina S, Omata M: Risk factors for recurring hepatocellular carcinoma differ according to infected hepatitis virus-an analysis of 236 consecutive patients with a single lesion. Hepatology 2000, 32:1216-23.

17. Chen CH, Huang GT, Yang PM, Chen PJ, Lai MY, Chen DS, Wang JD, Sheu JC: Hepatitis B- and C-related hepatocellular carcinomas yield different clinical features and prognosis. Eur J Cancer 2006, 42:2524-9.

18. Toyoda H, Kumada T, Kiriyama S, Sone Y, Tanikawa M, Hisanaga Y, Kanamori A, Yamaguchi A, Isogai M, Kaneoka Y, Washizu J: Characteristics and prognosis of patients in Japan with viral marker-negative hepatocellular carcinoma. J Gastroenterol Hepatol 2008, 23:459-66.

19. Higashi H, Matsumata T, Adachi E, Taketomi A, Kashiwagi S, Sugimachi K: Influence of viral hepatitis status on operative morbidity and mortality in patients with primary hepatocellular carcinoma. Br J Surg 1994 81:1342-5.

20. Higashi Y, Tada S, Miyase S, Hirota K, Imamura H, Kamio T, Suko H: Correlation of clinical characteristics with detection of hepatitis $B$ virus $X$ gene in liver tissue in HBsAg-negative, and HCV-negative hepatocellular carcinoma patients. Liver 2002, 22:374-9.

21. Huang $Y H$, Wu JC, Chen $C H$, Chang TT, Lee PC, Chau GY, Lui WY, Chang FY, Lee SD: Comparison of recurrence after hepatic resection in patients with hepatitis B vs. hepatitis C-related small hepatocellular carcinoma in hepatitis B virus endemic area. Liver Int 2005, 25:236-41.
22. Huo TI, Wu JC, Hsia CY, Chau GY, Lui WY, Huang YH, Lee PC, Chang FY, Lee SD: Hepatitis $C$ virus infection is a risk factor for tumor recurrence after resection of small hepatocellular carcinomas. World J Surg 2004, 28:787-91.

23. Yamanaka N, Takada M, Tanaka T, Yamanaka J, Yasui C, Ando T, Maeda S, Matsushita K, Okamoto E: Viral serostatus and coexisting inflammatory activity affect metachronous carcinogenesis after hepatectomy for hepatocellular carcinoma. A further report. J Gastroenterol 2000, 35:206-13.

24. Chen MF, Jeng LB, Lee WC, Chen TC: Surgical results in patients with dual hepatitis B- and C-related hepatocellular carcinoma compared with hepatitis B- or C-related hepatocellular carcinoma. Surgery 1998, 123:554-9.

25. Chen MF, Jeng LB, Lee WC: Surgical results in patients with hepatitis virus-related hepatocellular carcinoma in Taiwan. World J Surg 2002, 26:742-7.

26. Wu CC, Ho WL, Chen JT, Tang JS, Yeh DC, P'eng FK: Hepatitis viral status in patients undergoing liver resection for hepatocellular carcinoma. $\mathrm{Br} J$ Surg 1999, 86:1391-6.

27. Li Q, Li H, Qin Y, Wang PP, Hao X: Comparison of surgical outcomes for small hepatocellular carcinoma in patients with hepatitis $B$ versus hepatitis C: a Chinese experience. J Gastroenterol Hepatol 2007, 22:1936-41.

28. Nanashima A, Abo T, Sumida Y, Takeshita H, Hidaka S, Furukawa K, Sawai T, Yasutake T, Masuda J, Morisaki T, Nagayasu T: Clinicopathological characteristics of patients with hepatocellular carcinoma after hepatectomy: relationship with status of viral hepatitis. J Surg Oncol 2007, 96:487-92.

29. Cescon M, Cucchetti A, Grazi GL, Ferrero A, Viganò L, Ercolani G, Ravaioli M, Zanello M, Andreone P, Capussotti L, Pinna AD: Role of hepatitis B virus infection in the prognosis after hepatectomy for hepatocellular carcinoma in patients with cirrhosis: a Western dual-center experience. Arch Surg 2009, 144:906-13.

30. Takenaka K, Yamamoto K, Taketomi A, Itasaka H, Adachi E, Shirabe K, Nishizaki T, Yanaga $K$, Sugimachi $K$ : A comparison of the surgical results in patients with hepatitis B versus hepatitis C-related hepatocellular carcinoma. Hepatology 1995, 22:20-4.

31. Miyagawa S, Kawasaki S, Makuuchi M: Comparison of the characteristics of hepatocellular carcinoma between hepatitis B and C viral infection: tumor multicentricity in cirrhotic liver with hepatitis C. Hepatology 1996, 24:307-10.

32. Shiraishi M, Hiroyasu S, Nagahama M, Tomita S, Miyahira T, Kusano T, Furukawa M, Muto Y: Characteristics of hepatocellular carcinoma in patients with negative virus markers: clinicopathologic study of resected tumors. World J Surg 1999, 23:301-5.

33. Noguchi K, Nakashima O, Nakashima Y, Shiota K, Nawata H, Kojiro M: Clinicopathologic study on hepatocellular carcinoma negative for hepatitis $B$ surface antigen and antibody to hepatitis $C$ virus. Int J $\mathrm{Mol}$ Med 2000, 6:661-5.

34. Roayaie S, Haim MB, Emre S, Fishbein TM, Sheiner PA, Miller CM, Schwartz ME: Comparison of surgical outcomes for hepatocellular carcinoma in patients with hepatitis B versus hepatitis C: a western experience. Ann Surg Oncol 2000, 7:764-70.

35. Ahmad SA, Bilimoria MM, Wang X, Izzo F, Delrio P, Marra P, Baker TP, Porter GA, Ellis LM, Vauthey JN, Dhamotharan S, Curley SA: Hepatitis B or C virus serology as a prognostic factor in patients with hepatocellular carcinoma. J Gastrointest Surg 2001, 5:468-76.

36. Chen TH, Tseng LM, Chau GY, Lui WY, Tsay SH, King KL, Loong CC, Hsia CY, Wu CW: Clinicopathologic and prognostic differences between patients with hepatitis B- and C-related resectable hepatocellular carcinoma. $J$ Formos Med Assoc 2001, 100:443-8.

37. Wakai T, Shirai Y, Yokoyama N, Nagakura S, Hatakeyama K: Hepatitis viral status affects the pattern of intrahepatic recurrence after resection for hepatocellular carcinoma. Eur J Surg Oncol 2003, 29:266-71.

38. Yokoi Y, Suzuki S, Baba S, Inaba K, Konno H, Nakamura S: Clinicopathological features of hepatocellular carcinomas (HCCs) arising in patients without chronic viral infection or alcohol abuse: a retrospective study of patients undergoing hepatic resection. Gastroenterol 2005, 40:274-82

39. Uchiyama K, Ueno M, Hama T, Kawai M, Tani M, Terasawa H, Ozawa S, Uemura R, Nakase T, Yamaue H: Recurrence of primary hepatocellular 
carcinoma after hepatectomy-differences related to underlying hepatitis virus species. Hepatogastroenterology 2005, 52:591-5.

40. Sasaki Y, Yamada T, Tanaka H, Ohigashi H, Eguchi H, Yano M, Ishikawa O, Imaoka S: Risk of recurrence in a long-term follow-up after surgery in 417 patients with hepatitis B- or hepatitis C-related hepatocellular carcinoma. Ann Surg 2006, 244:771-80.

41. Kondo K, Chijiiwa K, Funagayama M, Kai M, Otani K, Ohuchida J: Differences in long-term outcome and prognostic factors according to viral status in patients with hepatocellular carcinoma treated by surgery. J Gastrointest Surg 2008, 12:468-76.

42. Kao WY, Su CW, Chau GY, Lui WY, Wu CW, Wu JC: A comparison of prognosis between patients with hepatitis $B$ and $C$ virus-related hepatocellular carcinoma undergoing resection surgery. World I Surg 2011, 35:858-67.

43. Chan HL, Sung JJ: Hepatocellular carcinoma and hepatitis B virus. Semin Liver Dis 2006, 26:153-61.

44. Castello G, Costantini S, Scala S: Targeting the inflammation in HCVassociated hepatocellular carcinoma: a role in the prevention and treatment. J Transl Med 2010, 8:109.

45. De Maria N, Manno M, Villa E: Sex hormones and liver cancer. Mol Cell Endocrinol 2002, 193:59-63.

46. Wang AG, Lee KY, Kim SY, Choi JY, Lee KH, Kim WH, Wang HJ, Kim JM, Park MG, Yeom YI, Kim NS, Yu DY, Lee DS: The expression of estrogen receptors in hepatocellular carcinoma in Korean patients. Yonsei Med J 2006, 47:811-6.

47. Chiu CM, Yeh SH, Chen PJ, Kuo TJ, Chang CJ, Chen PJ, Yang WJ, Chen DS: Hepatitis $B$ virus $\times$ protein enhances androgen receptor-responsive gene expression depending on androgen level. Proc Natl Acad Sci USA 2007, 104:2571-8, 20

48. Zhou Y, Sui C, Li B, Yin Z, Tan Y, Yang J, Liu Z: Repeat hepatectomy for recurrent hepatocellular carcinoma: a local experience and a systematic review. World J Surg Oncol 2010, 8:55.

49. Imamura H, Matsuyama $Y$, Tanaka E, Ohkubo T, Hasegawa K, Miyagawa S, Sugawara Y, Minagawa M, Takayama T, Kawasaki S, Makuuchi M: Risk factors contributing to early and late phase intrahepatic recurrence of hepatocellular carcinoma after hepatectomy. J Hepatol 2003, 38:200-7.

50. Squadrito G, Pollicino T, Cacciola I, Caccamo G, Villari D, La Masa T, Restuccia T, Cucinotta E, Scisca C, Magazzu D, Raimondo G: Occult hepatitis $B$ virus infection is associated with the development of hepatocellular carcinoma in chronic hepatitis C patients. Cancer 2006, 106:1326-30.

51. McKay A, Mackenzie S, Sutherland FR, Bathe OF, Doig C, Dort J, Vollmer CM $\mathrm{Jr}$, Dixon E: Meta-analysis of pancreaticojejunostomy versus pancreaticogastrostomy reconstruction after pancreaticoduodenectomy. Br J Surg 2006, 93:929-36.

52. Miao RY, Zhao HT, Yang HY, Mao YL, Lu X, Zhao Y, Liu CN, Zhong SX, Sang XT, Huang JF: Postoperative adjuvant antiviral therapy for hepatitis B/C virus-related hepatocellular carcinoma: a meta-analysis. World J Gastroenterol 2010, 16:2931-42.

doi:10.1186/1477-7819-9-108

Cite this article as: Zhou et al.: Influence of viral hepatitis status on prognosis in patients undergoing hepatic resection for hepatocellular carcinoma: a meta-analysis of observational studies. World Journal of Surgical Oncology 2011 9:108.

\section{Submit your next manuscript to BioMed Central and take full advantage of:}

- Convenient online submission

- Thorough peer review

- No space constraints or color figure charges

- Immediate publication on acceptance

- Inclusion in PubMed, CAS, Scopus and Google Scholar

- Research which is freely available for redistribution

Submit your manuscript at www.biomedcentral.com/submit
Biomed Central 